\title{
Comparaison des modèles mathématiques non linéaires et détermination du modèle qui décrit au mieux la croissance de la race Sardi
}

\author{
Mohammed Balafrej ${ }^{1 *}$ Abdelatif Sahnoun ${ }^{1}$ Mohamed Sadik ${ }^{2}$
}

\begin{abstract}
Mots-clés
Ovin, croissance, sélection, modèle mathématique, Maroc
\end{abstract}

\footnotetext{
Submitted: 3 September 2019

Accepted: 27 May 2020

Published: 1 December 2020

DOI: $10.19182 /$ remvt.31945
}

\begin{abstract}
Résumé
L'objectif de cette étude a été d'identifier le modèle mathématique non linéaire le plus approprié pour décrire la courbe de croissance de la race Sardi. Cette étude a été menée sur un troupeau d'ovins élevé dans la station de sélection de Krakra située à El Borouj au Maroc. Les effectifs comprenaient 763 mâles et 649 femelles correspondant respectivement à 3814 et 3240 observations pour les âges types : naissance, 10 jours, 30 jours, 90 jours et poids adulte. Pour modéliser la relation entre le poids et l'âge, cinq modèles ont été ajustés aux données de croissance, à savoir Brody, Logistic, Gompertz, Von Bertalanffy et Richards. Les modèles de croissance ont été ajustés aux données par la méthode du maximum de vraisemblance. Le critère $d^{\prime}$ information d'Akaike (AIC), le critère $d^{\prime}$ 'information bayésien (BIC) et la déviance ont été utilisés pour comparer la pertinence statistique des différents modèles de croissance. Parmi ces modèles, celui de von Bertalanffy a eu les plus petites valeurs d'AIC, du BIC et de la déviance, indiquant que ce modèle permettait la représentation des données la meilleure pour les deux sexes de cette race. Les paramètres de cette fonction peuvent être utilisés pour définir de nouveaux critères de sélection et améliorer les prévisions de production par une meilleure gestion de l'alimentation.
\end{abstract}

- Comment citer cet article : Balafrej M., Sahnoun A., Sadik M., 2020. Comparison of non-linear mathematical models and determination of the model that best describes the growth of the Sardi breed. Rev. Elev. Med. Vet. Pays Trop., 73 (4): 255-261, doi: 10.19182/remvt.31945

\section{INTRODUCTION}

Avec un effectif de 2,1 millions de têtes, la race Sardi constitue une des principales ressources génétiques ovines au Maroc (Boujnane, 2003). Elle a été décrite pour la première fois par Vaysse (1952), comme une race à lunettes, de grand gabarit, d'origine arabe, dont le berceau se situe dans les plateaux de l'Ouest marocain. La hauteur au garrot est presque d'un mètre et le poids adulte chez les mâles dépasse 100 kilogrammes. Ses caractéristiques morphologiques et ses performances de production la prédisposent principalement aux sacrifices

1. Direction de développement des filières de production, ministère de l'Agriculture, de la Pêche maritime, du Développement rural et des Eaux et Forêts, station D'bagh, avenue Hassan II, Rabat Maroc.

2. Ancien directeur de la station d'amélioration génétique ovine Krakra El Borouj, Maroc.

* Auteur pour la correspondance

Tél. : +212667444672 ; email : balafrejmed@hotmail.com religieux à un âge de plus de six mois et un poids variant de 40 à 70 kilogrammes (Boujnane, 2008). Il est à rappeler que la demande en ovins pour l'Aid El Adha est évaluée à environ cinq millions de têtes par an, ce qui représente environ $62 \%$ de la consommation de viandes ovines au Maroc (Anonyme, 2008).

L'amélioration génétique de cette race obéit à un schéma de sélection en deux étapes : une sélection phénotypique des produits mâles et femelles issus d'animaux identifiés inscrits au livre généalogique de la race, puis une sélection consistant à choisir les meilleurs produits sur la base du contrôle de performances par pesée à des âges types (naissance, 10, 30 et 90 jours) (Boujnane et al., 1995). Les principaux critères et les objectifs de sélection sont définis par le programme officiel de sélection génétique conduit par l'Association nationale ovine et caprine (ANOC) et concernent plus de 85000 femelles (ANOC, 2018).

Compte tenu des spécificités et de la destination de la Sardi, à savoir l'abattage pour les sacrifices religieux, il existe sûrement d'autres critères de sélection plus intéressants qui pourraient engendrer des progrès génétiques notables pour cette race (Boujnane, 2000). De 
nombreux travaux de caractérisation de la Sardi ont été conduits au Maroc (Boujnane et al., 2001) mais, à part l'étude de Janoune et al. (2015), aucun travail n'a été effectué sur la description et la caractérisation de la courbe de croissance de cette race. Janoune et al. (2015) concluent qu'une équation linéaire permet une représentation simple et néanmoins satisfaisante de la courbe de croissance durant la période avant sevrage, de telle sorte qu'il n'est pas nécessaire de recourir à des formes non linéaires, qu'ils jugent compliquées et difficiles à ajuster. La courbe de croissance est représentée mathématiquement comme une fonction reliant le poids vif à l'âge, couvrant tout ou partie de la durée de vie de l'animal (Daskiran et al., 2010). La croissance biologique peut être définie comme le gain de poids d'un animal jusqu'à ce qu'il atteigne l'âge adulte. Dans les premiers stades de la vie, cette croissance s'accélère et le gain de poids est supérieur à l'approche de l'âge adulte, produisant une courbe sigmoïde. Après le point d'inflexion, la croissance diminue progressivement et la vitesse de croissance devient de plus en plus lente. En biologie animale, les fonctions de croissance ont été utilisées depuis le début du $\mathrm{XX}^{\mathrm{e}}$ siècle. Les travaux clés dans le développement de ce concept sont ceux de Gompertz (1825), Brody (1945) et von Bertalanffy (1957).

Du point de vue économique, une croissance maximale pendant la période comprise entre la naissance et l'âge auquel l'animal est abattu pour la boucherie est généralement souhaitée. Cet âge est déterminé par des aspects économiques, comme la taille de la carcasse demandée par les consommateurs, mais aussi par des critères zootechniques comme le niveau d'engraissement dans la carcasse (Lupi et al., 2015). Cette phase de croissance varie d'une race à l'autre en fonction des aspects génétiques et environnementaux. Par ailleurs, l'âge d'abattage est affecté par les aspects culturels sur la base des différences de coutumes régionales.

Le cas de la Sardi fait donc exception à cette approche économique puisque les produits de cette race sont destinés à être abattus pour le sacrifice religieux à un âge avancé (au-delà de six mois) et un poids variant de 40 à 70 kilogrammes. La relation entre l'âge, la vitesse de croissance et la maturité de l'animal peut être étudiée à l'aide de ces modèles non linéaires à des fins de recommandations concernant la production, la sélection génétique et la reproduction. Ces modèles peuvent s'avérer d'une grande utilité pour définir de nouveaux critères de sélection de la Sardi en tenant compte de ses spécificités intrinsèques.

L'objectif de cette étude a été de comparer les modèles mathématiques non linéaires afin de déterminer le modèle qui décrit au mieux la croissance de la Sardi, notamment les performances pondérales, et de proposer des alternatives pour améliorer l'efficience du programme de sélection de la race. Les paramètres de la courbe peuvent être utilisés pour définir de nouveaux critères sélectifs, améliorer les prévisions de production par une meilleure gestion du calendrier alimentaire et faire correspondre l'alimentation à la production. Il faut noter que dans le cas des ateliers d'engraissement pour l'Aid Al Adha, la problématique est posée en termes de disponibilité des animaux dont le pic des naissances est fin automne début hiver, alors que l'Aid est mobile dans le temps selon le calendrier lunaire, ce qui pose des problèmes de prévision et de gestion des plans d'alimentation.

\section{MATERIEL ET METHODES}

Leétude a été conduite sur un troupeau d'ovins de race Sardi élevé à la station de sélection de Krakra située à El Borouj (32 $30^{\circ} \mathrm{N}$ et $7^{\circ} 11^{\prime} \mathrm{O}$ ) au sud de la province de Settat. Cette station est située dans un étage bioclimatique semi-aride avec une pluviométrie annuelle moyenne de 250 millimètres par an. Il est à noter que cette localisation correspond au berceau de cette race où le troupeau est conduit de manière extensive à l'instar de la majorité des élevages de la région.

La conduite alimentaire des troupeaux était basée sur le pâturage sur parcours pauvres et jachères, complémenté par un concentré à base de céréales et de tourteau de tournesol. Les performances de production des troupeaux ont été évaluées entre 1991 et 2001 à travers des pesées aux âges types naissance, 10 jours, 30 jours, 90 jours et 480 jours. Dans les effectifs se trouvaient 763 mâles et 648 femelles correspondant respectivement à 3814 et 3240 observations (pesées), soit au total 7054 observations (tableau I).

Les observations aberrantes ont été identifiées indépendamment pour chaque âge en utilisant l'intervalle interquartile qui est souvent utilisé pour trouver des valeurs aberrantes dans les données. Les valeurs aberrantes ont été définies comme des observations situées en dessous de Q1 - 1,5 IQR (Q1 : premier quartile; IQR : écart interquartile) ou au-dessus de Q3 + 1,5 IQR (Q3 : troisième quartile). Ce concept a été introduit pour la première fois en 1970 (Tukey, 1977 ; Wickham et Stryjewski, 2011). Après élimination des données aberrantes, le nombre d'observations a été réduit à 7018 observations (tableau I).

Par la suite, les individus ayant moins de quatre observations ont été retirés de la base de données ramenant ainsi le nombre total d'observations à 6998 correspondant à 1404 individus. Le tableau II montre les poids moyens aux âges types selon le sexe.

Pour modéliser la relation entre le poids et l'âge pour chaque sexe, cinq fonctions de croissance non linéaires ont été ajustées aux données de

Tableau I : Nombre d'observations (Obs) des ovins Sardi après suppression des observations aberrantes /// Number of observations (Obs) of Sardi sheep after removal of outliers

\begin{tabular}{|c|c|c|c|c|c|c|}
\hline & \multicolumn{2}{|c|}{$\begin{array}{l}\text { Situation de } \\
\text { départ }\end{array}$} & \multicolumn{2}{|c|}{$\begin{array}{c}\text { Après suppression } \\
\text { des Obs } \\
\text { aberrantes }\end{array}$} & \multicolumn{2}{|c|}{$\begin{array}{l}\text { Après suppressior } \\
\text { des individus } \\
\text { avec Obs }<4\end{array}$} \\
\hline & $\mathbf{N}$ & Obs & $\mathbf{N}$ & Obs & $\mathbf{N}$ & Obs \\
\hline Mâle & 763 & 3814 & 763 & 3789 & 757 & 3772 \\
\hline Femelle & 648 & 3240 & 648 & 3229 & 647 & 3226 \\
\hline Total & 1411 & 7054 & 1411 & 7018 & 1404 & 6998 \\
\hline
\end{tabular}

Tableau II : Nombre d'observations (Obs) des ovins Sardi et poids moyen aux âges types selon le sexe /// Number of observations (Obs) of Sardi sheep and average weight at typical ages by sex

\begin{tabular}{|c|c|c|c|c|c|c|c|c|c|c|}
\hline \multirow[t]{2}{*}{ Jour } & \multicolumn{2}{|c|}{0} & \multicolumn{2}{|c|}{10} & \multicolumn{2}{|c|}{30} & \multicolumn{2}{|c|}{90} & \multicolumn{2}{|c|}{480} \\
\hline & Mâle & Femelle & Mâle & Femelle & Mâle & Femelle & Mâle & Femelle & Mâle & Femelle \\
\hline Obs & 750 & 641 & 755 & 647 & 757 & 646 & 757 & 647 & 753 & 645 \\
\hline $\begin{array}{l}\text { Poids moyen } \\
(\mathrm{kg})\end{array}$ & $\begin{array}{c}4,065 \\
\pm 0,042\end{array}$ & $\begin{array}{c}3,819 \\
\pm 0,041\end{array}$ & $\begin{array}{c}6,372 \\
\pm 0,069\end{array}$ & $\begin{array}{c}5,951 \\
\pm 0,074\end{array}$ & $\begin{array}{r}10,715 \\
\pm 0,137\end{array}$ & $\begin{array}{c}9,998 \\
\pm 0,136\end{array}$ & $\begin{array}{l}25,177 \\
\pm 0,331\end{array}$ & $\begin{array}{l}22,689 \\
\pm 0,262\end{array}$ & $\begin{array}{l}66,372 \\
\pm 0,611\end{array}$ & $\begin{array}{c}48,722 \\
\pm 0,460\end{array}$ \\
\hline
\end{tabular}


croissance. Le tableau III montre les équations des modèles de croissance utilisés à savoir Brody, Logistic, Gompertz, von Bertalanffy et Richards.

L'interprétation biologique des paramètres de ces équations est la suivante : $A_{0}$ est le poids à la naissance $(t=0) ; A$ est le poids adulte ou à maturité représentant la limite asymptotique de chaque modèle, à mesure que l'âge $(t)$ s'approche de l'infini, c'est un poids moyen asymptotique (Brown et al., 1976) ; $B$ est une constante d'intégration qui n'a aucune interprétation biologique ; $K$ est la pente de la courbe de croissance et représente le taux de maturation postnatale ; une grande valeur de $K$ indique l'atteinte du poids adulte à un jeune âge ; et $M$ est le paramètre déterminant de la courbe du modèle de Richards (Malhado et al., 2009). La vitesse de croissance correspond à la dérivée $\mathrm{f}$ ' $(\mathrm{t})$ et le point d'inflexion correspond au point où la tangente traverse la courbe et où il y a changement de concavité. Dans ce cas, la dérivée seconde f''(t) s'annule en changeant de signe. L'âge d'inflexion est déduit à partir du point d'inflexion $\mathrm{f}[\mathrm{t}]$. La sélection faisant intervenir les points et âges d'inflexion répond aux objectifs de production des ovins destinés à l'abattoir ou la boucherie où le poids de carcasse se situe entre 15 et 17 kilogrammes et un âge d'environ cinq mois, ce qui n'est pas le cas pour la Sardi.

L'estimation préliminaire des paramètres $A, B, K$ et $M$ a été établie à l'aide de la procédure non linéaire NLIN de SAS correspondant au modèle de croissance à effets fixes, afin de déterminer les paramètres de départ du traitement de la procédure NLMIXED. Par la suite, une deuxième estimation a été réalisée avec le modèle à effets mixtes en utilisant cette procédure pour les deux sexes, qui prend en compte l'effet aléatoire associé aux individus. Cet effet aléatoire est dû au fait qu'à chaque individu correspondent potentiellement cinq enregistrements aux âges de pesées. La notation du modèle statistique non linéaire à effet mixte peut être représentée par $y i j=f\left(\psi i, x_{i j}\right)+g(\psi i$, $\left.\sigma, x_{i j}\right) \varepsilon_{i j}$ où $f$ représente le modèle structurel décrivant l'évolution du processus modélisé et la fonction $g$ le modèle d'erreur résiduelle, plus précisément sa variance, et peut dépendre de paramètres supplémentaires $\sigma$. Ce modèle suppose que la variabilité entre les sujets est représentée par le vecteur des paramètres individuels $\psi i$, et que les erreurs $\varepsilon_{i j}$ sont des échantillons aléatoires de la distribution normale centrée réduite, indépendantes de $\psi i$ (Comets et al., 2017).

Lorsque $f$ est non linéaire par rapport aux paramètres $\psi$, la notation décrit la forme générale des modèles à effets mixtes non linéaires. Les modèles ont été comparés en utilisant deux critères décrits ci-après.

Hirotugu Akaike a dérivé un estimateur de l'espérance (relative) de la distance de Kullback-Leibler basée sur la log-vraisemblance maximisée. Sa mesure, maintenant appelée critère d'information d'Akaike (Akaike information criterion ou AIC), a fourni un nouveau paradigme pour la sélection des modèles dans l'analyse des données empiriques (Burnham et al., 2002). L'AIC s'écrit : AIC $=2 k-2$ Ln L, où $k$ est le nombre de paramètres à estimer du modèle et $L$ le maximum de la fonction de vraisemblance du modèle. L'AIC est une mesure de la qualité d'un modèle statistique. C'est un critère de sélection de modèle basé sur la fonction de vraisemblance. Cette dernière quantifie la probabilité de réalisation ou d'occurrence des observations (i.e. des mesures de poids dans le jeu de données) sous l'hypothèse que le modèle statistique considéré a généré ces observations. Par conséquent, la maximisation de la fonction de vraisemblance détermine les paramètres qui sont le plus susceptibles de produire les données observées. Lorsque l'on estime un modèle statistique, l'ajout d'un paramètre au modèle augmente la vraisemblance de ce modèle. Considérer que le meilleur modèle est celui qui a la plus grande vraisemblance aboutirait à sélectionner un modèle contenant un grand nombre de paramètres. L'AIC permet de pénaliser les modèles en fonction du nombre de paramètres afin de satisfaire le critère de parcimonie. En effet, ce critère est une fonction décroissante de la vraisemblance du modèle et croissante du nombre de paramètres dans le modèle. Au sein d'un ensemble de modèles, on considère que celui qui a le plus petit AIC présente le meilleur compromis entre ajustement du modèle aux données (forte vraisemblance) et parcimonie du modèle (faible nombre de paramètres). Autrement dit, le critère du minimum d'AIC sélectionne un modèle qui explique relativement bien les données avec un nombre de paramètres relativement réduit.

Tableau III : Equations retenues pour les modèles de croissance des ovins Sardi et paramètres biologiques qui en découlent /// Equations retained for the Sardi sheep growth models and biological parameters derived from them

\begin{tabular}{|c|c|c|c|c|c|}
\hline Modèle & $\begin{array}{l}\text { Expression } \\
\text { mathématique } \\
\text { du modèle f(t) }\end{array}$ & Expression de B & Point d'inflexion & Age d'inflexion & $\begin{array}{l}\text { Vitesse de croissance } \\
\mathbf{f}^{\prime}(\mathbf{t})\end{array}$ \\
\hline Brody, 1945 & $A\left(1-B e^{-K t}\right)$ & $1-\left(\frac{A_{0}}{-}\right)$ & N'existe pas & N'existe pas & $K(A-f(t))$ \\
\hline Logistic ${ }^{*}$ & A & $(\mathrm{A})$ & A & $\ln B$ & $(f(t))$ \\
\hline & $\overline{\left(1+B e^{-K t}\right)}$ & $\left(\overline{A_{0}}\right)-1$ & $\overline{2}$ & $\overline{\mathrm{K}}$ & $\mathrm{Kf}(\mathrm{t})\left(1-\frac{}{\mathrm{A}}\right)$ \\
\hline Gompertz $^{* *}$ & $A e^{-B e^{-K t}}$ & $\ln \left(\frac{A}{A_{0}}\right)$ & $\frac{\mathrm{A}}{\mathrm{e}}$ & $\frac{\ln B}{K}$ & $K f(t) \ln \left(\frac{A}{f(t)}\right)$ \\
\hline Von Bertalanffy, 1957 & $A\left(1-B e^{-K t}\right)^{3}$ & $1-\left(\frac{A_{0}}{A}\right)^{3}$ & $\frac{8}{27} \mathrm{~A}$ & $\frac{\ln 3 B}{K}$ & $3 \mathrm{Kf}(\mathrm{t})^{\frac{2}{3}}\left(\mathrm{~A}^{\frac{1}{3}}-\mathrm{f}(\mathrm{t})^{\frac{1}{3}}\right)$ \\
\hline Richards, 1959 & $A\left(1-B e^{-K t}\right)^{M}$ & $1-\left(\frac{A_{0}}{A}\right)^{M}$ & $\mathrm{~A}\left(1-\frac{1}{\mathrm{M}}\right)^{\mathrm{M}}$ & $\frac{\ln M B}{K}$ & $M K f(t)^{\left(1-\frac{1}{M}\right)}\left(A^{\frac{1}{M}}-f(t)^{\frac{1}{M}}\right)$ \\
\hline \multicolumn{6}{|c|}{ 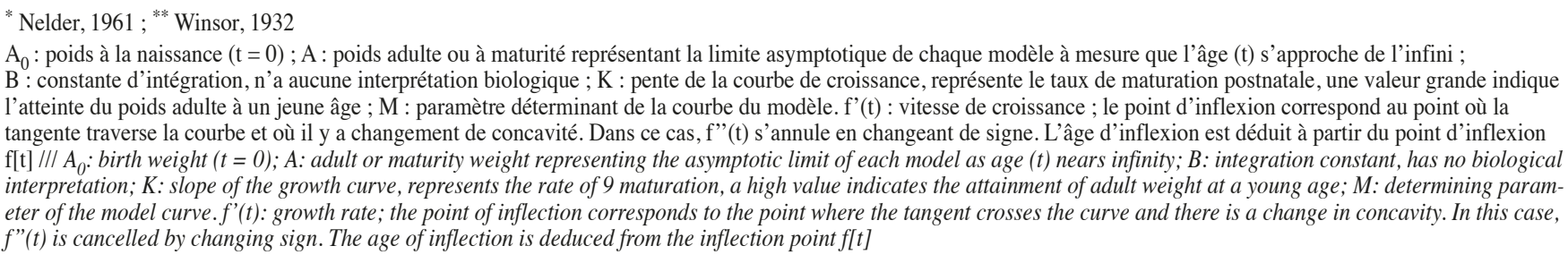 } \\
\hline
\end{tabular}


Par conséquent, le choix se porte sur le modèle avec l'AIC le plus faible (Cameron et Trivedi, 2005).

Le critère d'information bayésien (BIC) est dérivé de l'AIC et proposé par Schwarz (1978) : BIC $=-2 \operatorname{Ln}(L)+k \operatorname{Ln}(N)$ avec $L$ la vraisemblance du modèle estimée, $N$ le nombre d'observations dans l'échantillon et $k$ le nombre de paramètres libres du modèle. A la différence d'AIC, la pénalité dépend de la taille de l'échantillon et non pas seulement du nombre de paramètres. Comme pour l'AIC, on choisit alors le modèle avec le BIC le plus faible.

\section{RESULTATS ET DISCUSSION}

\section{Performances pondérales}

Les poids moyens aux âges types et par sexe sont rapportés au tableau II. Ils étaient élevés par rapport à ceux de la littérature pour la même race mais similaires à ceux obtenus par Chikhi et Boujenane (2003) dans des conditions d'élevage intensif où les animaux étaient conduits en stabulation permanente avec une alimentation basée sur le foin de luzerne et un mélange d'aliments concentrés. A l'opposé, le troupeau de cette étude a été conduit en élevage extensif basé sur le pâturage à l'instar de la conduite prédominante de la région.

\section{Comparaison des différents modèles de croissance}

Les paramètres $A, B, K$ et $M$ ont été estimés pour les modèles de courbe de croissance non linéaire étudiés, ainsi que les AIC, BIC et -2 Log-Likelihood (-2 Log-L) (tableau IV). En se basant sur ces critères le modèle de von Bertalanffy présente les valeurs de l'AIC, du BIC et de -2 Log-L (déviance) les plus faibles, même si les valeurs de ces critères pour les autres modèles ne sont pas très éloignées du premier modèle. Ainsi, le modèle de von Bertalanffy semble décrire au mieux la courbe de croissance pour les deux sexes de la race Sardi (tableau IV ; figures 1 à 4). Ce résultat concorde avec les modèles développés par Lupi et al. (2015) et Topal et al. (2004) travaillant respectivement sur la race espagnole Segureña et la race Awassi en

Tableau IV : Paramètres d'ajustement des modèles aux données de croissance des ovins Sardi selon le sexe /// Parameters for fitting models to Sardi sheep growth data by sex

\begin{tabular}{|c|c|c|c|c|c|c|c|c|c|c|}
\hline \multirow[t]{2}{*}{ Paramètre } & \multicolumn{2}{|c|}{ Brody } & \multicolumn{2}{|c|}{ Logistic } & \multicolumn{2}{|c|}{ Gompertz } & \multicolumn{2}{|c|}{ Von Bertalanffy } & \multicolumn{2}{|c|}{ Richards } \\
\hline & Mâle & Femelle & Mâle & Femelle & Mâle & Femelle & Mâle & Femelle & Mâle & Femelle \\
\hline A & 87,4503 & 52,8896 & 66,8731 & 48,7166 & 67,9963 & 49,047 & 69,8392 & 49,5884 & 71,6003 & 49,8447 \\
\hline B & 0,9539 & 0,9307 & 11,6268 & 8,9131 & 2,6559 & 2,4132 & 0,6001 & 0,5642 & 0,8165 & 0,7439 \\
\hline K & 0,00308 & 0,00508 & 0,02174 & 0,02295 & 0,01104 & 0,01288 & 0,008273 & 0,01017 & 0,00629 & 0,00858 \\
\hline M & $* * *$ & $* * *$ & $* * *$ & $* * *$ & $* * *$ & $* * *$ & $* * *$ & $* * *$ & 1,6789 & 1,8699 \\
\hline-2 Log-L & 19069 & 15272 & 17490 & 13722 & 16092 & 12477 & 15394 & 11927 & 19178 & 13881 \\
\hline $\mathrm{AIC}$ & 19089 & 15292 & 17510 & 13742 & 16112 & 12497 & 15414 & 11947 & 19208 & 13911 \\
\hline $\mathrm{BIC}$ & 19135 & 15336 & 17556 & 13787 & 16158 & 12542 & 15460 & 11992 & 19277 & 13978 \\
\hline
\end{tabular}

A : poids adulte ou à maturité $; \mathrm{B}$ : constante d'intégration $; \mathrm{K}$ : pente de la courbe de croissance $; \mathrm{M}$ : paramètre déterminant de la courbe du modèle ; AIC : critère d'information d'Akaike; BIC : critère d'information bayésien I/I A: adult or mature weight; B: integration constant; K: slope of the growth curve; M: determining parameter of the model curve; AIC: Akaike information criterion; BIC: Bayesian information criterion

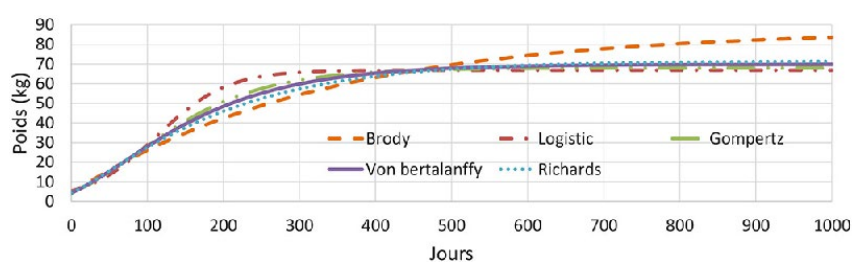

Figure 1 : Courbe de croissance des béliers Sardi selon les modèles retenus /// Growth curve of Sardi rams according to the selected models

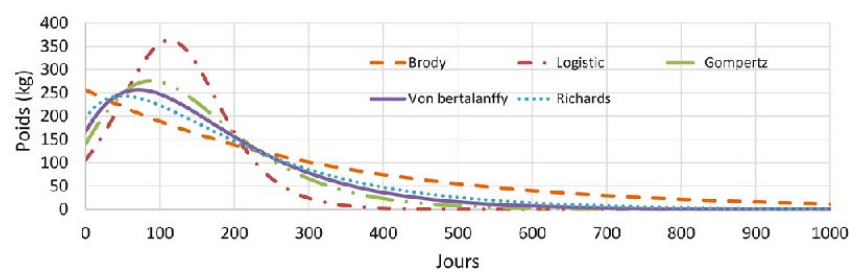

Figure 2 : Evolution de la vitesse de croissance des béliers Sardi selon les modèles retenus /// Evolution of the growth rate of Sardi rams according to the selected models

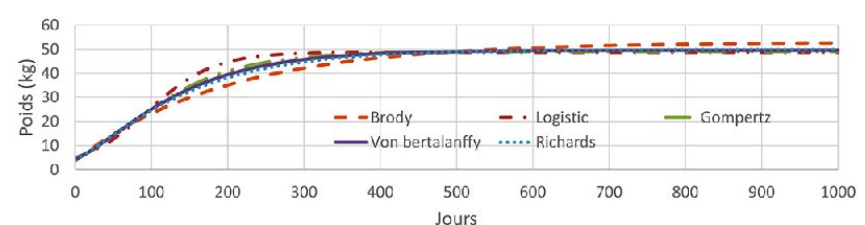

Figure 3 : Courbe de croissance des brebis Sardi selon les modèles retenus /// Growth curve of Sardi ewes according to the selected models

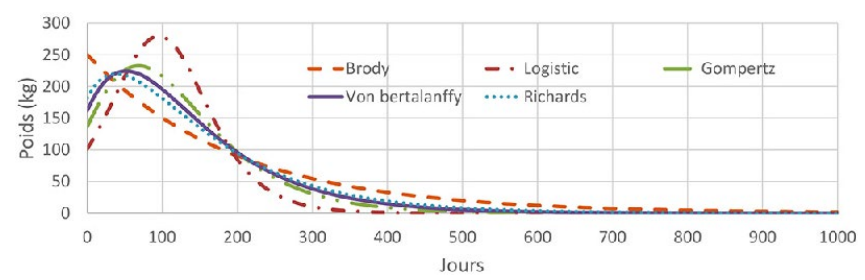

Figure 4 : Evolution de la vitesse de croissance des brebis Sardi selon les modèles retenus /// Evolution of the growth rate of Sardi ewes according to the selected models 
Turquie. Enfin, Pittroff et al. (2008), travaillant sur des données de croissance d'un troupeau de la race Targhee aux Etats-Unis, affirment que selon les critères précités le modèle de von Bertalanffy est dans la majorité des cas le mieux indiqué pour décrire la croissance de la race précitée.

Par ailleurs, d'autres études ont conclu qu'une meilleure description de la croissance des races ovines locales pourrait être effectuée par d'autres modèles comme celui de Gompertz pour les agneaux Morkaraman en Turquie (Topal et al., 2004) et le modèle Logistic pour la Santa Inês au Brésil (Silva et al., 2012) et la Norduz en Iran (Daskiran et al., 2010). Aussi, le modèle de Gompertz était le mieux approprié pour décrire la croissance des Nordus (Kum et al., 2010) ainsi que des Scottish Blackface et Texel (Lambe et al., 2006). Pour la race ovine brésilienne Morada Nova, Souza et al. (2013) trouvent que les modèles Meloun I, III et IV (non considérés dans la présente étude), ayant la plus faible valeur de l'AIC et de BIC, montrent une supériorité par rapport aux autres modèles. Pour leur part Gbangboche et al. (2008) trouvent que le modèle de Brody donne la plus faible valeur de l'AIC et semble bien traduire la courbe de croissance de la race Dwarf de l'Ouest africain. Enfin, Behzadi et al. (2014) trouvent que la fonction Brody permet le meilleur ajustement pour les deux sexes de la race Balouchi en Iran. Le tableau $\mathrm{V}$ propose une synthèse bibliographique après ajustement des données de croissance des différentes races ovines aux modèles non linéaires.

Dans la présente étude, l'estimation de la constante $A$ qui est le poids adulte ou à maturité des mâles (correspondant à 480 jours) a varié selon les modèles. Pour le modèle retenu de von Bertalanffy ce paramètre était proche de $69,8 \mathrm{~kg}$. Comparé aux autres races, il paraissait largement supérieur à ceux du Texel $(40 \mathrm{~kg})$ et du Scottish Blakface (35 kg) rapportés par Lambe et al. (2006), et inférieur à celui de la Segureña (77,5 kg) selon Lupi et al. (2015). Pour les femelles, le poids adulte estimé a été pour la Sardi de $49,7 \mathrm{~kg}$, alors que pour la femelle Segureña il a été de 49,6 kg, traduisant ainsi l'effet de la race et du système d'élevage sur ce paramètre.

\section{Recherche des points d'inflexion}

Brody (1945) estime que le point d'inflexion de la courbe de croissance coïncide avec le début de la puberté. Cette conclusion est basée sur la modélisation des données de croissance à partir de différentes espèces avec un modèle non linéaire. Selon ce concept, la période de croissance d'un animal est divisée en deux phases distinctes : la phase autoaccélérée (prépubertaire) et la phase auto-inhibante (postpubertaire). En considérant que le modèle de von Bertalanffy donnait la meilleure estimation de la courbe de croissance de la Sardi, le point d'inflexion pour cette race se situait autour de 71 jours pour les mâles et 52 jours pour les femelles, pour des poids respectifs de 20,7 et $14,7 \mathrm{~kg}$ (tableau VI ; figure 5). Ces chiffres sont très proches des valeurs relevées par Lupi et al. (2015) pour la Segureña, (respectivement 70,4 et 56,6 jours, et 20,6 et 14,5 kg). Cependant, et en désaccord avec la loi de Brody (1945), Pittroff et al. (2008) affirment qu'il n'existe aucune relation entre les paramètres de la courbe de croissance et les indicateurs physiologiques comme la puberté, car leurs travaux montrent que la puberté intervient plus tardivement que le point d'inflexion de la courbe de croissance.

\section{CONCLUSION}

Les cinq modèles non linéaires, à savoir Brody, Logistic, Gompertz, von Bertalanffy et Richards, ont été ajustés aux données de croissance de la Sardi par la méthode du maximum de vraisemblance. Parmi ces modèles, la fonction de von Bertalanffy avait les plus petites valeurs des critères AIC, BIC et de déviance, indiquant une

Tableau VI : Points d'inflexion de $\mathrm{f}(\mathrm{t})$ selon le sexe des ovins Sardi selon les modèles /// Inflection points of $f(t)$ by sex of Sardi sheep according to models

\begin{tabular}{lcccc} 
Modèle & \multicolumn{2}{c}{ Mâle } & \multicolumn{2}{c}{ Femelle } \\
\cline { 2 - 5 } & $\mathbf{t}_{\mathbf{i}}$ (jours) & $\left.\mathbf{f}\left(\mathbf{t}_{\mathbf{i}}\right) \mathbf{( k g}\right)$ & $\mathbf{t}_{\mathbf{i}}$ (jours) & $\left.\mathbf{f}\left(\mathbf{t}_{\mathbf{i}}\right) \mathbf{( k g}\right)$ \\
\hline Brody & - & - & - & - \\
Logistic & 112,8 & 33,4 & 95,3 & 24,4 \\
Gompertz & 88,5 & 25,0 & 68,4 & 18,0 \\
Von Bertalanffy & 71,1 & 20,7 & 51,7 & 14,7 \\
Richards & 50,1 & 15,7 & 38,5 & 11,9
\end{tabular}

Tableau $\mathbf{V}$ : Données de la littérature après ajustement des données de croissance de différentes races ovines aux modèles non linéaires /// Literature data after fitting growth data from different sheep breeds to non-linear models

\begin{tabular}{|c|c|c|c|c|c|c|c|}
\hline \multirow[t]{2}{*}{ Race } & \multirow[t]{2}{*}{ Pays } & \multirow[t]{2}{*}{ Sexe } & \multirow[t]{2}{*}{ Modèle } & \multicolumn{3}{|c|}{ Paramètre } & \multirow[t]{2}{*}{ Auteur } \\
\hline & & & & A & B & K & \\
\hline \multirow[t]{2}{*}{ Segureña } & \multirow[t]{2}{*}{ Espagne } & M & Von Bertalanffy & 67,707 & 0,628 & 0,009 & \multirow[t]{2}{*}{ Lupi et al., 2015} \\
\hline & & $\mathrm{F}$ & Von Bertalanffy & 48,877 & 0,587 & 0,01 & \\
\hline Locale & Pakistan & $M$ et $F$ & Von Bertalanffy & 38,95 & 0,48 & 0,2 & Aman Ullah et al., 2013 \\
\hline Awassi & \multirow[t]{2}{*}{ Turquie } & $\mathrm{F}$ & Von Bertalanffy & 41,7 & 0,52 & 0,01 & \multirow[t]{2}{*}{ Topal et al., 2004} \\
\hline Morkaraman & & $\mathrm{F}$ & Gompertz & 41,4 & 2,06 & 0,012 & \\
\hline \multirow[t]{2}{*}{ Norduz } & \multirow[t]{2}{*}{ Turquie } & M & Logistic & 51,3 & ND & ND & \multirow[t]{2}{*}{ Daskiran et al., 2010} \\
\hline & & $\mathrm{F}$ & Logistic & 47,2 & ND & ND & \\
\hline Naine Ouest africaine & Bénin & $M$ et $F$ & Brody & 46,9 & 0,962 & 0,0024 & $\begin{array}{c}\text { Gbangboche et al., } \\
2008\end{array}$ \\
\hline \multirow[t]{2}{*}{ Baluchi } & \multirow[t]{2}{*}{ Iran } & M & Brody & 47,62 & 0,92 & 0,007 & \multirow[t]{2}{*}{ Behzadi et al., 2014} \\
\hline & & $\mathrm{F}$ & Brody & 43,45 & 0,92 & 0,007 & \\
\hline
\end{tabular}

$\mathrm{M}$ : mâle ; F : femelle. A : poids adulte ou à maturité ; B : constante d'intégration ; K : pente de la courbe de croissance /// M: male; F: female. A: adult or mature weight; B: integration constant; $K$ : slope of the growth curve 


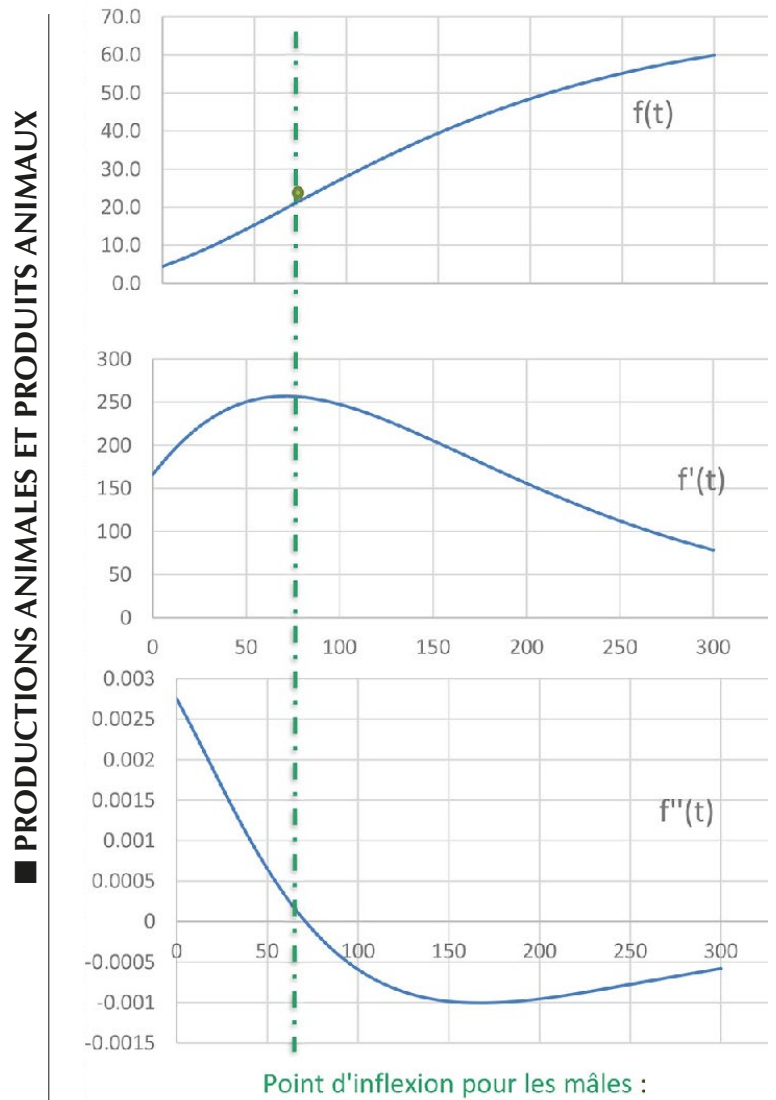

$f(t)=20.7 \mathrm{~kg} \mathrm{n} ; \mathrm{ti}=71.1$ jours

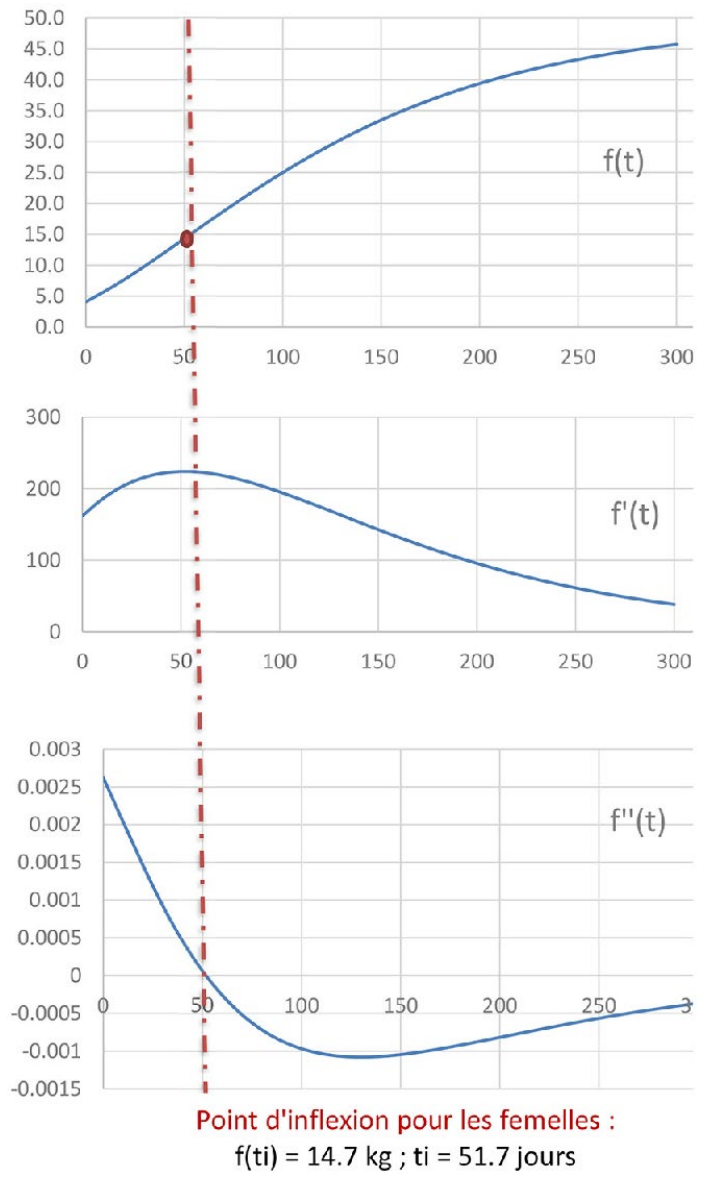

Figure 5 : Représentations graphiques du modèle de von Bertalanffy $f(t)$ et ses dérivées pour les béliers et les brebis Sardi /// Graphical representations of the von Bertalanffy model $f(t)$ and its derivatives for Sardi rams and ewes meilleure représentation des données par ce modèle que par les autres modèles considérés pour les deux sexes de cette race. Les paramètres de cette fonction peuvent être utilisés pour définir de nouveaux critères de sélection et améliorer les prévisions de production par une meilleure gestion de l'alimentation. Les critères de sélection doivent être adaptés pour sélectionner des animaux donnant les meilleures performances de croissance à l'abattage tardif. En effet, le poids au point d'inflexion peut correspondre au poids d'abattage si le poids de maturité de la race est élevé (paramètre $A$ ) ou coïncidant avec une demande du marché orientée vers les carcasses légères. Ceci est le cas pour la Segureña en Espagne pour laquelle les travaux de Lupi et al. (2015) montrent que son poids d'abattage correspond au point d'inflexion de cette race. Par contre, dans le cas de la race Sardi le point d'inflexion est loin de l'objectif d'abattage visant des carcasses lourdes. Par conséquent, il est recommandé de retenir un point dans la courbe de croissance qui s'approcherait de l'âge et du poids d'abattage de cette race, susceptible d'être utilisé dans la sélection de cette race. En effet, il est à rappeler que le poids au point d'inflexion pour le modèle de von Bertalanffy représente environ $30 \%$ du poids asymptotique. Afin de répondre à l'objectif de sélection spécifique à cette race, il est suggéré de déterminer un point de caractérisation situé au-delà du point d'inflexion. En outre, l'adaptation individuelle au modèle de von Bertalanffy montre que les tendances de performance pour certains animaux de la base de données de cette étude peuvent être inversées avec l'évolution de l'âge, ce qui pourrait pénaliser certains individus si on se base uniquement sur le point d'inflexion.

La présente étude devrait être aussi poursuivie par un travail sur des données récentes recueillies sur des animaux issus du troupeau de la station de sélection génétique de Krakra. Le but serait d'évaluer l'importance du progrès de sélection réalisé par les programmes de sélection génétique de la race.

\section{Déclaration des contributions des auteurs}

MB et AS ont participé à la conception de l'étude, l'analyse, l'interprétation des données et la rédaction de la première version du manuscrit ; MS a procédé à la collecte des données ; tous les auteurs ont participé à la révision critique du manuscrit dont ils ont approuvé la forme actuelle.

\section{Conflits d'intérêts}

Les auteurs déclarent que l'étude a été réalisée sans conflit d'intérêts.

\section{REFERENCES}

ANOC, 2018. Principales races ovines et caprines sélectionnées au Maroc. Association nationale ovine et caprine, Rabat, Maroc

Anonyme, 2008. Situation prévisionnelle d'approvisionnement du marché des animaux ; Direction de l'Elevage, Ministère de l'Agriculture, Rabat, Maroc

Behzadi B.M.R., Aslaminejad A.A., Sharifi A.R., Simianer H., 2014. Comparison of Mathematical models for describing the growth of Baluchi sheep. J. Agr. Sci. Tech., 14: 57-68

Boujenane I., Ait Bihi N., Boukallouche A. 1995. Amélioration génétique des ovins au Maroc. In: Gabiña D. (ed.). Strategies for sheep and goat breeding. Zaragoza: CIHEAM, 109-119, (Cahiers Options Méditerranéennes; n. 11)

Boujnane I., 2000. Séminaire de I'AMPA sur l'amélioration génétique au service de l'élevage ovin, Rabat, Maroc, 24-25 Nov. 2000

Boujnane I., 2008. Technique d'engraissement des agneaux. Transfert de technologie, Bulletin mensuel d'information et de liaison du PNTTA, Rabat, Maroc

Boujnane I., M'Zian S., Sadik M., 2001. Estimation des paramètres génétiques et phénotypiques de la croissance des ovins de race Sardi. Actes Inst. Agron. Vét. (Maroc), 21 (3): 177 
Brody S., 1945. Bioenergetics and Growth with special reference to the efficiency complex in domestic animals. V. 1044, Hafner publishing company, New York, USA, 499 p.

Brown J.E., Fitzhugh Jr. H.A., Cartwright T.C., 1976. A Comparison of Non Linear Models for Describing Weightage Relationships in Cattle. J. Anim. Sci., 42: 810-818, doi: 10.2527/jas1976.424810x

Burnham K.P., Anderson D.R., 2002. Model Selection and Inference: A Practical Information-Theoretic Approach. 2nd Edition, V 488, Springer-Verlag, New York, USA, 60-61

Chikhi A., Boujnane I., 2003. Characterization of the Sardi Sheep Breed in Morocco. Rev. Elev. Med. Vet. Pays. Trop., 56 (3-4): 187-192, doi : 10.19182/remvt.9863

Cameron A.C., Trivedi P.K., 2005. Microeconometrics: Methods And Applications, Cambridge University Press, USA, 1056 p, doi: 10.1017/ CBO9780511811241

Daskiran I., Koncagul S., Bingol M., 2010. Growth caracteristics of indigenous Norduz female and male lambs. J. Agric. Sci., 16: 62-69, doi: 10.1501/ Tarimbil_0000001122

Comets E., Lavenu A., Lavielle M., 2017. Parameter Estimation in Nonlinear Mixed Effect Models Using saemix, an R Implementation of the SAEM Algorithm. J. Stat. Softw., 80 (3), doi: 10.18637/jss.v080.i03

Gbangboche A.B., Glele-Kakai R., Salifou S., Albuquerque L.G., Leroy P.L., 2008. Comparaison of non-linear growth models to describe the growth curve in West African Dwarf sheep. Anim: Internat. J. Anim. Biosci., 2: 1003-1012, doi: 10.1017/S1751731108002206

Gompertz B., 1825. On the nature of the function of the law of human mortality, and on a new mode of determining the value of life contingencies. Phil. Trans. R. Soc., 115: 513-583, doi: 10.1098/rstl.1825.0026

Janoune A., Boujnane I., Derqaoui L., 2015. Genetic analysis of live weight of sardi sheep using random regression and multi-trait animal models. Small Rumin. Res., 130: 1-7, doi: 10.1016/j.smallrumres.2015.06.015

Kum D., Karakus K., Ozdemir T., 2010. The best non-linear function for body weight at early phase of Norduz femal lambs. Trakia J. Sci., 8 (2): 62-67

Lambe N.R., Navajas E.A., Simm G., Bünger L., 2006. A genetic investigation of various growth models to describe growth of lambs of two contrasting breeds. J Anim. Sci., 84 (10): 2642-2654, doi: 10.2527/jas.2006-041

\section{Summary}

Balafrej M., Sahnoun A., Sadik M. Comparison of non-linear mathematical models and determination of the model that best describes the growth of the Sardi breed

The objective of this study was to identify the most appropriate non-linear mathematical model to describe the growth curve of the Sardi breed. This study was conducted on a flock of sheep reared in the Krakra breeding station located in El Borouj, Morocco. The flock included 763 males and 649 females corresponding respectively to 3814 and 3240 observations for the following typical ages: birth, 10 days, 30 days, 90 days and adult weight. To model the relationship between weight and age, five models were fitted to the growth data, i.e. Brody, Logistic, Gompertz, von Bertalanffy, and Richards. The growth models were fitted to the data using the maximum likelihood method. The Akaike information criterion (AIC), Bayesian information criterion (BIC) and deviance were used to compare the statistical relevance of the different growth models. Among these models, von Bertalanffy's function had the lowest values of $\mathrm{AIC}, \mathrm{BIC}$ and deviance, indicating that this model provided the best representation of data for both sexes of this breed. The parameters of this function can be used to define new selection criteria and improve production predictions through better feed management.

Keywords: sheep, growth, selection, mathematical models, Morocco
Lupi T.M., Nogales S., Leon J.M., Barba C., Delgado V. 2015. Characterization of commercial and biological growth curves in the Segureña sheep breed. Animal, 1-8, doi: 10.1017/S1751731115000567

Malhado C.H.M., Carneiro P.L.S., Affonso P.R.A.M., Souza A.A.O., Sarmento J.L.R., 2009. Growth curves in Dorper sheep with crossed with the local Brazilian Breeds, Morada Nova, Rabo Largo, and Santa Inês. Small Rumin. Res., 84 (1-3): 16-21, doi: 10.1016/j.smallrumres.2009.04.006

Nelder J.A., 1961. The fitting of a generalization of the logistic curve, Biometrics, 17, 89-110, doi: 10.2307/2527498

Pittroff W., Dahm F., Keisler D., Cartwright T.C., 2008. Onset of puberty and the inflection point of the growth curve in sheep-Brody's law revisited. J. Agric. Sci., 146 (3): 239-250, doi: 10.1017/S0021859608007806

Richards F.J., 1959. A flexible growth function for empirical use, J. Exp. Bot., 10: 290-300, doi: 10.1093/jxb/10.2.290

Schwarz G., 1978. Estimating the dimension of model. Ann. Statist., 6 (2): 461-464, doi: 10.1214/aos/1176344136

Silva L.S.A., Fragaa A.B., Silva F.L., Beelena P.M.G., Silva R.M.O., Tonhatic H., Barrosa C.C., 2012. Growth curve in Santa Inês sheep. Small Rumin. Res. 105 (1-3): 182-185, doi: 10.1016/j.smallrumres.2011.11.024

Souza L.A., Carneiro P.L.S., Malhado C.H.M., Silva F.F., Silveira F.G., 2013. Traditional and alternative nonlinear models for estimating the growth of Morada Nova sheep. Rev. Bras. Zootec., 42 (9) : 651-655, doi: 10.1590/ S1516-35982013000900007

Topal M., Ozdemir M., Aksakal V., Dogru U., 2004. Determination of the best non-linear function in order to estimate growth in Morkaraman and AwAssi lambs. Small Rumin. Res., 55 (1-3): 229-232, doi: 10.1016/j.smallrumres.2004.01.007

Tukey J.W., 1977. Exploratory Data Analysis. Addison-Wesley Publishing Company Reading, Mass. - Menlo Park, Cal., London, Amsterdam, Don Mills, Ontario, Sydney 1977, XVI, 688 S. doi: 10.1002/bimj.4710230408

Vaysse J., 1952. L'élevage au Maroc. Presse des imprimeries Réunies, Casablanca, Maroc

Von Bertalanffy L., 1957. Quantitative laws in metabolism and Growth. Quart. Rev. Biol., 32 (3), doi: 10.1086/401873

Wickham H., Stryjewski L., 2011. 40 years of boxplots. 17 p.

Winsor C.P., 1932. The Gompertz curve as a growth curve. Proc. Natl. Acad. Sci., 18 (1): 1-8, doi: 10.1073/pnas.18.1.1

\section{Resumen}

Balafrej M., Sahnoun A., Sadik M. Comparación de los modelos matemáticos no lineares y determinación del modelo que mejor describe el crecimiento de la raza Sardi

El objetivo del presente estudio fue el de identificar el modelo matemático no linear más apropiado para describir la curva de crecimiento de la raza Sardi. Este estudio se llevó a cabo en un hato de ovinos criados en la estación de selección de Krakra, situada en Borouj, en Marruecos. Los efectivos incluyeron 763 machos y 649 hembras correspondiendo respectivamente a 3812 y 3240 observaciones para las siguientes edades tipo: nacimiento, 10 días, 30 días, 90 días y peso adulto. Para modelizar la relación entre el peso y la edad, se ajustaron cinco modelos a los datos de crecimiento, es decir Brody, Logistic, Gompertz, von Bertalanffy y Richards. Los modelos de crecimiento se ajustaron a los datos mediante el método de máxima similitud. El criterio de información de Akaike (AIC), el criterio de información bayesiano (BIC) y la desviación fueron utilizados para comparar la pertinencia estadística de los diferentes modelos de crecimiento. Entre estos modelos, la función de von Bertalanffy presentó los valores más pequeños de $\mathrm{AIC}$, de BIC y de la desviación, indicando que este modelo permitió la mejor representación de los datos para los dos sexos de esta raza. Los parámetros de esta función pueden ser utilizados para definir nuevos criterios de selección y mejorar las previsiones de producción para una mejor gestión de la alimentación.

Palabras clave: ovinos, crecimiento, selección, modelos matemáticos, Marruecos 
